\title{
The Internet of Chemical Things
}

\section{Steven V. Ley, Daniel E. Fitzpatrick, Richard J. Ingham and Nikzad Nikbin}

The Internet of Things (IoT), a term first coined by Ashton in 1999 [1], concerns the interconnection and effective networking of machines and computing devices through the infrastructure of the internet. Yet simply stated like this, it does not do justice to what is becoming a revolutionary phenomenon in terms of how our electronic-based physical objects (Things) will communicate and interact into the future. It is expected that within five years there will be around 26 billion devices wirelessly connected through the Internet [2], with some predictions saying many more [3].

The digital world and the impact of IoT in industry and society are transforming how we live our lives today. The applications are widespread and touch us all; from healthcare, energy management and energy storage to environmental monitoring and global transportation of goods, materials for IoT devices are coming under closer scrutiny. Furthermore, with the sheer volumes of information produced by IoT applications, data security and protection are of utmost importance [7]. In the era of connectivity, it is vital that personal privacy be cherished, given that it is so hard earned.

Nevertheless, the world is technologically and scientifically evolving rapidly and we must engage responsibly rather than simply remaining witnesses to that change. The concepts of the IoT go way beyond simple machine-to-machine (M2M) communication [8]. New systems evolve and adapt through sharing of networks. Advanced innovative software, neural networks and machine learning techniques lead to intelligent IoT systems making connections and creating value across a variety of disciplines and diverse areas.

\section{$6 \mathbf{6} \quad$ The beginning of the Internet of Chemical Things (IoCT), which we define as the interconnection and networking of chemical machines, computing devices and all chemical services delivered through the infrastructure of the Internet, is emerging.}

IoT is set to revolutionise how the world operates. Not only the homes, but even whole cities of the future [4] and the vast majority of our consumer products and functional materials [5] will be influenced by IoT. However, like all disruptive technology it is not without controversy. Many rightly fear the proliferation of self-adapting systems and loss of intellectual property [6]. With the world focussed increasingly on sustainability, the sources for
From a chemistry perspective, the IoT has yet to make a significant impact on our science. This is perhaps not too surprising, as while chem-

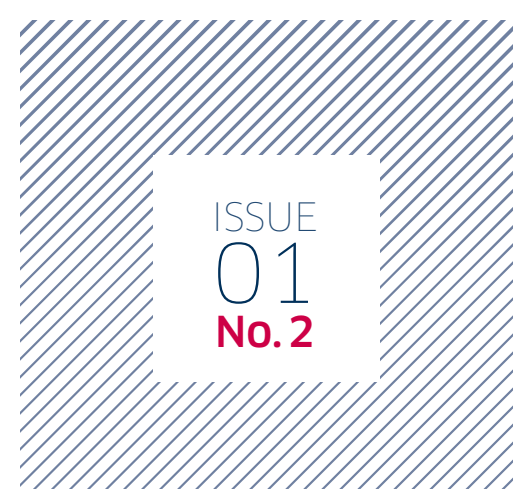

ists are visionary thinkers, they are often conservative in their approach and only like to propose what they can realistically deliver. Nevertheless, the beginnings of the Internet of Chemical Things (IoCT), which we define as the interconnection and networking of chemical machines, computing devices and all chemical services delivered through the infrastructure of the Internet, is emerging. This holistic approach can be expected to deliver efficiencies and greatly improved chemical resource management.

Given our own particular research interests in the development of flow chemistry methods and continuous processing [9], we have been increasingly drawn into confronting the challenges presented by using a machine-assisted approach to synthesis [10,11]. The dynamic nature of flow chemistry, particularly multi-step telescoped processing, inevitably leads to the development of a wide skill set including the need to look at the system as a whole. This is important for the development of self-optimising systems where feedback and control algorithms are paramount. Enhanced visualisation techniques such as the use of high speed and thermal imaging cameras can be exploited to go beyond human eyesight [12-14]. A number of further projects have benefited from the ability to use devices in an interconnected manner, including the integration of FAC-MS biological screening with multi-step flow chemical synthesis. Access to data is equally important in these cases, whether this be for real-time access to experimental parameters and conditions, or for interpretation of analytical data to direct a compound's design, to carry out synthesis and evaluate its activity.

A new generation of small, low-power computer systems are positioned to play a key role in the 


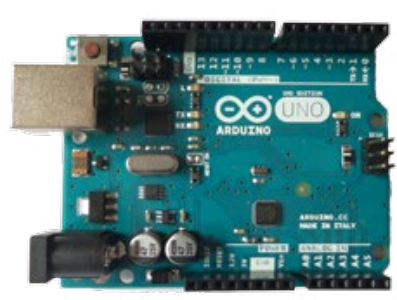

development of the IoCT (Scheme 1). For example, the Arduino, a microcontroller prototyping board, is used for inexpensive sensors and robotics applications. More powerful systems such as the Raspberry Pi, Intel Galileo, Intel Edison and Beaglebone are better suited to more intensive tasks, including data processing and detailed calculations.

The advent of these new devices presents new challenges when they are used in a science environment. Factors such as size, energy consumption and processing power, traditionally the domain of electrical and electronic engineers, must be taken into account by researchers performing reactions. This highlights the pressing need for chemists to become familiar with and to adopt a holistic systems approach when designing experiments. No longer is it acceptable to just think of a reaction outcome - factors such as solvent choice, rate of product formation, environmental impact and reagent expense have entered into a chemist's thinking; now it is time for technology to do the same. We firmly believe that the IoCT devices will facilitate this transition of thought and provide better continuity across disciplines, resulting in obvious synergistic benefits.

So how exactly will the IoCT impact the chemistry community? Further to the greater control strategies described above - themselves leading to improved laboratory safety and flexibility in chemists' working regimes - we can expect increased data collection to lead to the discovery of new reactivity patterns. Small changes in parameters (for example temperature, $\mathrm{pH}$ or colour) that previously may have been missed or deemed

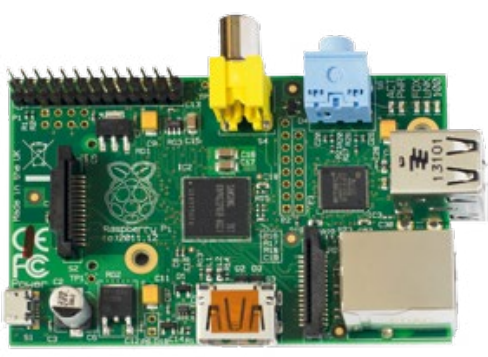

unimportant will now be recorded. The ability to amalgamate recording systems with analytical capabilities such as IR and MS will improve our understanding of molecular processes. By integrating these capabilities with the processing power of the cloud, time consuming data analysis tasks will be relegated to machines and advanced machine-learning algorithms will perform pattern analysis to find potential data series of interest. Ultimately, chemists will be released from repetitive tasks, enabling them to spend more time planning future experiments and greatly increasing productivity.

Machines and intelligent control systems are changing the way research is conducted in both academia and industry. Many examples exist emphasising how the use of such tools has positively influenced the chemistry community. The Internet of Chemical Things is poised to alter further the research landscape for the better. We believe it is time to protect our precious human resource by allowing our machines a degree of autonomy to assist our future intellectual development. In the next few years chemistry will change in the ways outlined above; it is important that the community not only accept this change but welcome it with open arms, so as to benefit from the full advantages that technological advancement brings.

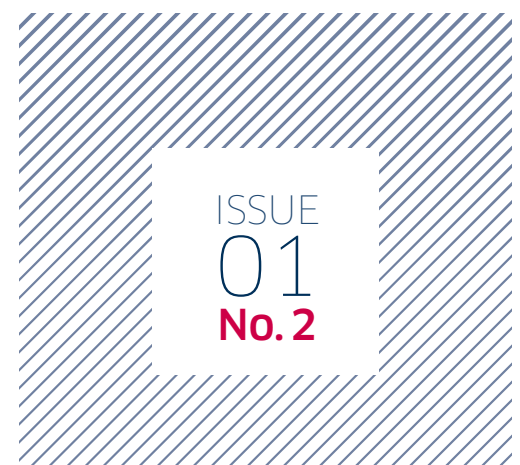

Scheme 1. Small low-power computer systems can play a key role in the Internet of Chemical Things. The Arduino (left) is used for inexpensive sensor and robotics applications. More powerful systems such as Raspberry Pi (right) are better suited for data processing and calculations.

Authors

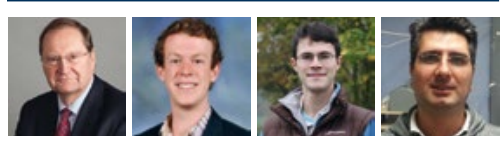

Steven V. Ley

Director of Research at the Department of Chemistry

University of Cambridge, Lensfield Road, Cambridge CB2 1EW, UK

凹svl1000@cam.ac.uk

Daniel E. Fitzpatrick

PhD Student

Richard J. Ingham

PhD Student

Nikzad Nikbin

Post-doctoral Research Associate

\section{Selected References}

For the complete list of references please see the online publication.

9. Refer to the Ley Group website for our publications related to flow chemistry and continuous processing, http://www.leygroup.ch.cam.ac.uk/

10. Ingham, R. J.; Battilocchio, C.; Fitzpatrick, D. E.; Sliwinski, E.; Hawkins, J. M.; Ley, S. V. Angew. Chem., Int. Ed. Engl. 2015, 54, 144-148. doi:10.1002/ anie.201409356

11. Ingham, R.J.; Battilocchio, C.; Hawkins, J.M.; Ley, S.V. Beilstein J. Org. Chem. 2014, 10, 641-652. doi:10.3762/ bjoc.10.56

\section{Copyright}

doi: 10.3762/bmag.2.

(c) 2015 The author(s). Published by Beilstein-Institut. This is an open access article under the CC BY license (http://creativecommons.org/licenses/ by/4.0). This article is part of Issue 1 of the Beilstein Magazine. Copyright by Beilstein-Institut.

www.beilstein-institut.de

Published online 16/10/2015 